\title{
Identification of Circular RNAs Altered in Mouse Jejuna After Radiation
}

\author{
Qianying Lu Wei Gong Jinhan Wang Kaihua Ji Yan Wang Chang Xu \\ Yang Liu Ningning He Liqing Du Qiang Liu \\ Institute of Radiation Medicine, Chinese Academy of Medical Sciences \& Peking Union Medical \\ College, Tianjin Key Laboratory of Molecular Nuclear Medicine, Tianjin, China
}

\section{Key Words}

Radiation-induced intestinal injury $•$ CircRNAs $•$ Sequencing

\begin{abstract}
Background/Aims: Circular RNAs (circRNAs) make up a large class of non-coding RNAs and play important roles in a variety of diseases, including nervous system diseases and cancers. The intestinal epithelium is sensitive to ionizing radiation, radiotherapy of abdominal or pelvic tumors or nuclear accident exposure can lead to high radiation toxicity, which can result in radiation-induced intestinal injury. The goal of this present study was to analyze the potential roles of circRNAs in radiation-induced intestinal injury. Methods: Mice were divided into two groups: control group and irradiated group. Irradiated group was 3.5 days after 14Gy abdominal irradiation (ABI) group. We started with RNA-seq of circRNA changes in mouse jejuna after radiation and validated by RT-PCR in the following experimental. miRNAs targeted mRNAs were predicted using proprietary software based on target scan and Miranda. The network of circRNA-miRNA-mRNA was illustrated by cytoscape software. Results: 2751 circRNAs were detected in the two groups. At day 3.5 post-radiation, 42 and 48 circRNAs were found to be significantly upregulated and downregulated, respectively, compared to the control $(p \leq 0.05$, Fold Change 22). Further, the altered expression of 10 circRNAs (chr18:35610871-35613502+, chr15:95864225-95894541+, chr3:96041338-96042928-, chr5:64096979-64108263+, chr19:16705875-16710941-, chr5:134491893-134500149-, chr19:42562552-42564341+, chr5:32640331-32664400+, chr3:72958113-72960367- and chr8:79343654-79372364-) were verified by RT-PCR. Compared the miRNA-targeted mRNAs with our mRNAs sequencing data, we found 14 upregulated circRNA-targeted mRNAs were also unregulated and 22 downregulated circRNAs-targeted mRNAs were also downregulated. Gene ontology and KEGG pathway analyses indicated the predicted genes were mainly involved in the MAPK signaling pathway. Conclusions: This study reveals that expression of circRNAs was altered in the jejuna of mice post-irradiation and provides a resource for the study of circRNAs in radiation-induced intestinal injury and repair.




\section{Introduction}

Circular RNAs (circRNAs) are a large class of non-coding RNAs. Unlike traditional linear RNAs that contain 5' and 3' ends, circRNAs arise from 3' -5 ' ligations of the ends of linear RNA molecules [1]. Due to the absence of $5^{\prime}$ and $3^{\prime}$ ends, circRNAs are more stable than linear RNAs and resistant to RNase R [2,3]. A significant majority of circRNAs are exonic circRNAs [4]. Models of formation for these circRNAs include lariat-driven circularization [5] and intron-pairing driven circularization, the latter of which also generates exonicintronic circRNAs (EIciRNAs). The generation of intronic circRNAs (ciRNAs) usually involves a consensus motif containing a 7-nt GU-rich element near the 5' splice site and an 11-nt C-rich element near the branchpoint [6]. In addition, some circRNAs also form through RNAbinding protein or trans-factor driven circularization [7].

These circRNAs have a wide distribution and are found in many species, including humans [8], mice [2], Drosophila [9], and fish [10]. Biological functions have been reported for circRNAs, where functions have been most widely studied for microRNAs (miRNAs) sponges. The circRNAs and messenger RNAs (mRNAs) contain shared miRNA response elements, where the circRNAs regulate gene expression through miRNA binding. For example, human CDR1as, which is mainly located in the brain, has over $70 \mathrm{miR}-7$ binding sites [11] and has been proposed to function as a sponge for miR-7 by reducing the amount of free miR-7 molecules and, consequently, influence brain functions associated with neuropsychiatric disorders. Certain ciRNAs [6] and EIciRNAs [12] are abundant in nuclei, where ciRNAs interact with the polymerase II machinery, EIciRNAs interact with U1 small nuclear ribonucleoproteins, and both modulate the expression of parental gene in a cis-acting manner. A subset of circRNAs can combine with proteins and regulate protein function and a few circRNAs can be translated.

Recent work has suggested that circRNAs play important roles in disease development. The circRNA CDR1as is highly expressed in the brain and has been implicated in nervous system diseases, including Parkinson's and Alzheimer's disease [13], and brain development. In some cancers, such as colon [14], liver [15], and stomach cancers [16], circRNA expression differs from expression in corresponding normal tissue, indicating a role for these circRNAs in tumor development. Radiotherapy is used to treat more than half of cancer patients and plays a critical role in curing $25 \%$ of cancers [17]. While radiotherapy does play an important role in cancer treatment, normal tissue surrounding the cancerous tissue also suffers from radiation damage [18]. The intestinal epithelium is sensitive to ionizing radiation and the intestine is one of the most sensitive organs to radiation toxicity [19]. Radiotherapy of abdominal and pelvic tumors and exposure to nuclear accidents can result in high radiation toxicity that can lead to radiation-induced intestinal injury. Radiationinduced bone marrow injury can be alleviated using bone marrow transplantation, however, there are no effective means of preventing or treating radiation-induced intestinal injury [20]. Radiation-induced intestinal toxicity is typically defined as clonogenic cell death and apoptosis of crypt cells, insufficient replacement of villus epithelium, breakdown of the mucosal barrier, and significant inhibition of compensatory proliferation [21]. The effects of toxicity on the intestines are collectively referred to as gastrointestinal acute radiation syndrome [22], which clinically manifests with symptoms including anorexia, vomiting, diarrhea, dehydration, systemic infection, and, in extreme cases, septic shock and death [23].

Researchers have reported on approaches to preventing radiation-induced intestinal injury. Gong [24] et al. found that mesenchymal stem cells (MSCs) can improve the rate of survival of mice suffering from radiation poisoning. After irradiating the abdomen with 14 Gy, administration of mesenchymal stem cells increases the rate of survival to $20 \%$ compared to $0 \%$ survival in the control PBS group. Liang [25] found that 12-0-tetradecanoylphorbol13-acetate can improve survival after radiation by increasing intestinal crypt stem cell survival via acting through the PKC signaling pathway. Others have also found that improving differentiation of goblet cells [26] or preventing endothelial cell dysfunction [27] can 
Lu et al.: Jejual CircRNAs Altereation After Radiation

also alleviate radiation-induced intestinal injury. However, as mentioned above, there are currently no recognized means of preventing or treating radiation-induced intestinal injury.

Due to the high tissue and developmental stage specificity of circRNAs, these molecules are regarded as potential disease biomarkers. As circRNAs are widely expressed in vivo, we believe that there are circRNAs expressed in the intestine that play an important role in radiation-induced intestinal injury and repair. According to a previous report, mice suffer the most severe intestinal injury at 3.5 days post-radiation and recover by day 5 through intestinal repair. Therefore, sequencing of mouse jejuna was performed at 3.5 days postradiation, in order to identify circRNAs associated with radiation-induced injury and protection.

\section{Materials and Methods}

\section{Mouse model of radiation-induced intestinal injury}

Male C57BL/6 mice, aged 8-10 weeks and weighing 20-22 g, were purchased from Huafukang Biotechnology Co. Ltd (Beijing, China). All mice were housed in a temperature-controlled and specificpathogen-free environment with a $12 \mathrm{~h}$ light/dark cycle and fed standard chow and water ad libitum. Abdominal irradiation was performed using a Cs137 $\gamma$-ray irradiator (Atomic Energy of Canada, Chalk River, Ontario, Canada), where lead shielding was used to protect other parts of the body from irradiation. Mice were exposed to $14 \mathrm{~Gy}$ at a rate of $1 \mathrm{~Gy} / \mathrm{min}$ at room temperature. Day 3.5 post-radiation, the mice were sacrificed and their jejuna harvested and frozen at $-80^{\circ} \mathrm{C}$. All experimental procedures and protocols were conducted in accordance with the guidelines of the local animal care and use committee.

\section{Samples and RNA isolation}

The mice were divided into control group and irradiated group. The control group contained three non-irradiated mice and the irradiated group contained three mice exposed to $14 \mathrm{~Gy}$ abdominal irradiation. RNA was isolated from the jejuna of the control group and irradiation group at 3.5 days post-radiation using TRizol reagent (Ambion) according to the manufacturer's instructions. The sample RNA concentrations were measured at an OD260/280 using a NanoDrop ND-2000 instrument (Thermo, Waltham, MA, USA). The integrity of the RNA in the samples was assessed using denaturing agarose gel electrophoresis.

\section{High-throughput sequencing}

High-throughput whole transcriptome sequencing and subsequent bioinformatics analysis were performed by Cloud-Seq Biotech (Shanghai, China) as follows. First, rRNA was removed from the total RNA using a Ribo-Zero rRNA Removal Kit (Illumina, USA) according to the manufacturer's instructions. Library Construction RNA libraries were constructed using a TruSeq Stranded Total RNA Library Prep Kit (Illumina, USA) from the rRNA-depleted RNA according to the manufacturer's instructions. RNA quality and quantity in the libraries were controlled for using the BioAnalyzer 2100 system (Agilent Technologies, USA). Libraries were denatured into single-stranded DNA molecules, captured on Illumina flow cells, amplified in situ as clusters, and sequenced for 150 cycles on an Illumina HiSeq Sequencer according to the manufacturer's instructions.

\section{Sequencing analysis of circRNA}

Paired-end reads were obtained from the Illumina HiSeq 4000 sequencer. After 3' adaptor-trimming and removal of low quality reads using cutadapt [28] software (v1.9.3). The resulting high-quality trimmed reads were used to analyze circRNA. The high-quality reads were aligned to the reference genome/ transcriptome using STAR [29] software and circRNAs were identified using DCC [30] software and annotated from the circBase [31] database.

\section{Identification of differentially expressed circRNAs}

Edger [32] software was used to normalized the data and analyze circRNAs for differential expression. Any circRNAs exhibiting fold-changes $\geq 2.0$ with P-values $\leq 0.05$ were considered significantly differentially expressed.

\section{KARGER}




\begin{tabular}{|c|c|c|}
\hline $104 y$ & Cell Physiol Biochem 2018; & $17: 2558-2568$ \\
\hline and Biochemistry & $\begin{array}{l}\text { DOI: } 10.1159 / 000491652 \\
\text { Published online: July } 10,2018\end{array}$ & $\begin{array}{l}\text { (O) } 2018 \text { The Author(s). Published by S. Karger AG, Basel } \\
\text { www.karger.com/cpb }\end{array}$ \\
\hline
\end{tabular}

Lu et al.: Jejual CircRNAs Altereation After Radiation

\begin{tabular}{|c|c|c|c|c|}
\hline \multirow{2}{*}{$\begin{array}{l}\text { Experimental validation of } \\
\text { circRNAs }\end{array}$} & \\
\hline & Gene Name & Primer Type & Primer Sequence & Product Length \\
\hline Quantitative & chr18:35610871-35613502+ & $\begin{array}{l}\text { Forward primer } \\
\text { Reverse primer }\end{array}$ & $\begin{array}{l}\text { ACGCTGTTTCCAAGAAATGC } \\
\text { ACTGCTGCGACTTGGATCTT }\end{array}$ & 195 \\
\hline time PCR (qRT-PCR) & chr15:95864225-95894541+ & $\begin{array}{l}\text { Forward primer } \\
\text { Reverse primer }\end{array}$ & $\begin{array}{l}\text { CAGAGGCGAATCGACTTCAT } \\
\text { GGGCTTCCCGTTAAATTCTT }\end{array}$ & 188 \\
\hline used to validate circRNA & chr3:9 & Forward primer & CAAAGCACGTGACAGTGGTC & 197 \\
\hline expression. Of the circRNAs & chr5:64096979-64108263 & Forward primer & $\begin{array}{l}\text { GAAACAGAGCCCAACAGCTC } \\
\text { GAAACAGACACA }\end{array}$ & 204 \\
\hline identified, 5 upregulated & & $\begin{array}{l}\text { Reverse primer } \\
\text { Forward primer }\end{array}$ & $\begin{array}{l}\text { CATTCGAGAAATCCCTGCTC } \\
\text { TCACGACCCTGGATAACACA }\end{array}$ & \\
\hline downregulated & $\mathrm{r} 1 \mathrm{~T}$ & Reve & TTGAGGA & 169 \\
\hline & chr5:134491893-134500149- & $\begin{array}{l}\text { Forward primer } \\
\text { Reverse primer }\end{array}$ & $\begin{array}{l}\text { CCACAAACAGGAGGTGATCG } \\
\text { TGGGATGTCAAGGCTGTCAC }\end{array}$ & 126 \\
\hline & $\operatorname{chr} 19: 4$ & $\begin{array}{l}\text { Forward primer } \\
\text { Reverse primer }\end{array}$ & $\begin{array}{l}\text { GGAAGTAGTGCTGTGGAGGAA } \\
\text { GGCACCACTTCAATCTCTAGT }\end{array}$ & 95 \\
\hline 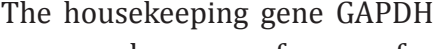 & chr5:32640331-32664400+ & Forward primer & $\begin{array}{l}\text { TGAGG } \\
\text { TCATC }\end{array}$ & 113 \\
\hline was used as a & chr3:72958113-7296036 & $\begin{array}{l}\text { Reverse primer } \\
\text { Forward primer }\end{array}$ & $\begin{array}{l}\text { CCATTATCAAATCTGGATATGGGCAC } \\
\text { GAATCCAGCAGCTGACACA } \\
\text { TGGCTTTAAGTCAAGACTTTGTTGT }\end{array}$ & 103 \\
\hline normalization. The primers were & & $\begin{array}{l}\text { Reverse primer } \\
\text { Forward primer }\end{array}$ & $\begin{array}{l}\text { TGGCTTTAAGTCAAGACTTTGTTGT } \\
\text { TACTGGCGCAGTCTGTGAAC }\end{array}$ & 201 \\
\hline designed using $\mathrm{tl}$ & chr8:79343654-79372364- & Reverse primer & CGCAGAGCCAACAGAGATTT & 201 \\
\hline strategy, where & Gapdh & $\begin{array}{l}\text { Forward primer } \\
\text { Reverse primer }\end{array}$ & $\begin{array}{l}\text { AGGTCGGTGTGAACGGATTTG } \\
\text { GGGGTCGTTGATGGCAACA }\end{array}$ & 95 \\
\hline
\end{tabular}

was amplified (Table 1). Total

RNA was reverse-transcribed

into complementary DNA using a PrimeScript RT Reagent Kit (Perfect Real Time; TaKaRa, Osaka, Japan) and subjected to qRT-PCR on an Applied Biosystems 7500 Fast Real-Time PCR System. Three independent assays were performed for all samples, where samples were assessed in triplicate. The relative expression of the circRNAs was calculated using the $2-\Delta \Delta \mathrm{Ct}$ method.

Gene ontology and KEGG pathway analysis of selected circRNAs

Gene ontology (GO) and KEGG analyses were performed for differentially expressed circRNA-associated genes. GO analysis assessed molecular functions, biological processes, and cellular components. The top 10 enriched GO terms among the two groups were highlighted as differentially expressed. KEGG pathway analysis was performed to identify pathways associated with circRNAs targeted mRNAs..

\section{Analysis of circRNA-miRNA-mRNA network}

Recent studies have reported that circRNAs can precisely regulate miRNA levels by absorbing miRNA and, thus, acting as "miRNA sponge." To explore the functions of circRNAs, as well as deduce which circRNAs function as miRNA sponges, predictions of interactions of differentially expressed circRNAs with miRNAs were made using popular miRNA target gene prediction software.

The miRNA binding sites and target mRNAs were predicted using proprietary software based on targetscan and Miranda. Using each circRNA with its miRNA-binding and predicted miRNA sites as data, cytoscape software was used to construct a circRNA-miRNA-mRNA network map and, thus, visualize interactions between these molecules.

\section{Results}

Expression pattern of circRNAs in mouse jejuna after exposure to radiation

A total of 2751 circRNAs were identified in mice jejuna at baseline and 3.5 days postradiation based on at least one read spanning a head-to-tail splice junction. Of these, 1809 circRNAs were already included in the circBase or had been identified in previous studies, while 942 circRNA were first observed in this present study.

The 2751 circRNAs were found to be located in genes across all chromosomes (Fig. 1A). Chromosomes 1-19 contained more than 80 circRNAs each and X had 42 circRNAs, while the $\mathrm{Y}$ and mitochondrial chromosomes contained less than 10 circRNAs. There were 2418 exonic circRNAs, which ranged in size from 80 nt to more than $2000 \mathrm{nt}(3.34 \%)$ and most (14.36\%) were 301-400 nt long (Fig. 1B), the average length was $732.58 \mathrm{nt}$.

Although most of the genes (67.70\%) generated a single circRNA, 553 genes generated two or more circRNAs. Among these, $222(13.97 \%)$ of genes generated more than two circRNAs and some genes (1.93\%) generated more than 6 circRNAs (Fig. 1C). As presented in Fig. 1D, these 2751 circRNA were from a variety of regions, including exonic $(87.90 \%)$, 
Fig. 1. Expression of circRNAs in jejuna of mice after exposure to radiation. (A) Chromosomal distribution of circRNAs. "ChrM" represents mitochondrial genome. (B) Length distribution of 2418 exonic circRNAs. The majority of circRNAs ranged from 301-400 nt (16.34\%) in size. (C) Distribution of circRNAs per gene, where most genes contained one circRNA. (D) Host genes from which these 2751 circRNAs derived.

sense overlapping (9.16\%), and intronic regions $(2.51 \%)$, based on their location in their host genes.

Identification of differentially expressed circRNAs after exposure to radiation

Among the 2751 circRNAs, 1970 circRNAs were detected in the control group, 1845 circRNAs were detected at day 3.5 post-radiation, and 1064 circRNAs were detected in both groups (Fig. 2A). Of these, 90 circRNAs differentially expressed at 3.5 days post-radiation were selected, where 42 were upregulated and 48 downregulated (Fig. 2B). Based on the data presented in Table 2, the miRNAs and miRNAtarget mRNAs associated with these circRNAs were identified using our mRNA sequencing results. As shown in Fig. 2C, 14 upregulated circRNAtargeted upregulated mRNAs and 22 downregulated circRNA-targeted downregulated mRNAs (Table 3) were subjected to GO and KEGG pathway analyses to characterize the circRNA-miRNA-mRNA network.

\section{Validation of circRNA expression by $q R T-P C R$}

To validate the expression levels of differentially expressed circRNAs, 5 upregulated and 5 downregulated circRNAs from day 3.5 post-radiation were selected and expression levels were confirmed by qRT-PCR (Fig. $3)$. The qRT-PCR results for the upregulated and downregulated circRNAs were consistent with the

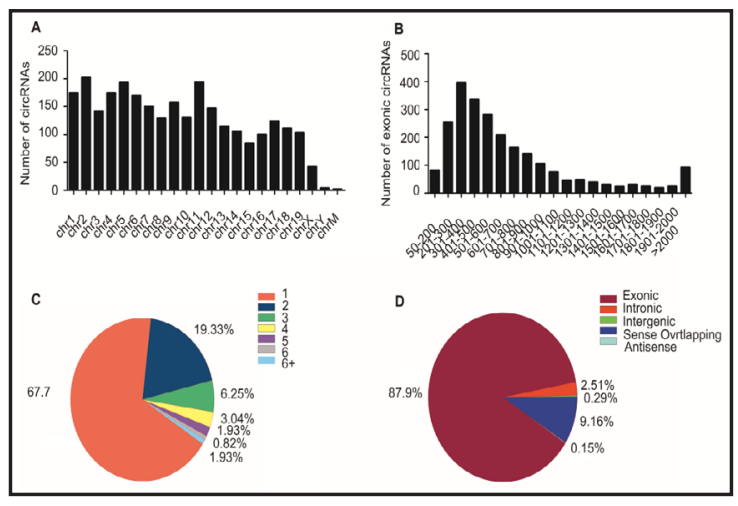

Table 2. 90 differentially expressed circRNAs at 3.5 days post-radiation

\begin{tabular}{|c|c|c|c|c|c|c|}
\hline AID & $\log$ & $\log C P M$ & PV & Regulation & A Type & Gene Symbol \\
\hline Ir15:99794409-99807743- & 4.45612 & 10.160646 & 0.0100157 & Up & Exonic & Lima1 \\
\hline chr6:88355483-88358642- & 2.5512206 & 10.365014 & 0.0449825 & Up & Exonic & Eefsec \\
\hline chr5:100399347-100408113- & 3.6381245 & 10.191793 & 0.0335328 & Up & Exonic & Sec31a \\
\hline chr4:132857300-132871657- & 3.8963318 & 10.003852 & 0.0442012 & Up & Exonic & Stx12 \\
\hline chr14:55659200-55660098- & 3.9533835 & 10.191841 & 0.0200185 & Up & Exonic & Mdp1 \\
\hline chr14:27438599-27442620+ & 3.6243888 & 10.273047 & 0.028087 & Up & Exonic & Fam208a \\
\hline chr19:43810471-43814814+ & 3.2565184 & 10.382725 & 0.0480682 & Up & Exonic & Abcc2 \\
\hline & 3.9583763 & 10.715693 & 0.0099943 & Up & Exonic & Lrp6 \\
\hline chr11:58040391-58044050+ & 3.6411373 & 10.053388 & 0.0465824 & Up & Exonic & Larp1 \\
\hline chr6:22132229-22145687+ & 3.6634433 & 10.151658 & 0.03783 & Up & Exonic & Cped1 \\
\hline $\begin{array}{l}\text { chr19:41545875-41555701+ } \\
\text { chry.90793296-900763680+ }\end{array}$ & $\begin{array}{r}2.7124662 \\
2800458\end{array}$ & 10.361739 & 0.0307496 & Up & Exonic & Lcor \\
\hline $\begin{array}{l}\text { chrrY:907932966-90793680+ } \\
\text { chr6-86943357-86967373+ }\end{array}$ & $\begin{array}{l}2.8010458 \\
3.6637903\end{array}$ & $\begin{array}{l}10.619317 \\
10.18469\end{array}$ & 0.0050547 & Up & Exonic & Erdr1 \\
\hline $\begin{array}{l}\text { chr6:86944357-86967373+ } \\
\text { chr15:95864225-95894541+ }\end{array}$ & $\begin{array}{l}3.6637903 \\
3.9069187\end{array}$ & $\begin{array}{l}10.184692 \\
10.094897\end{array}$ & $\begin{array}{l}0.0332887 \\
0.0300941\end{array}$ & $\begin{array}{l}\text { Up } \\
\text { Up }\end{array}$ & $\begin{array}{l}\text { Exonic } \\
\text { Exonic }\end{array}$ & $\begin{array}{l}\text { Aak1 } \\
\text { Ano6 }\end{array}$ \\
\hline chrY:90793395-90793680+ & 4.1790799 & 10.101849 & 0.0204181 & Up & Sense overlapping & Erdr1 \\
\hline chrY:90793325-90793680+ & 4.7300929 & 10.358742 & 0.0018511 & Up & ense overlapping & Erdr1 \\
\hline chr3:116618256-116621508+ & 4.1544319 & 10.145074 & 0.0183458 & $\mathrm{Up}$ & Exonic & Sass6 \\
\hline chr4:58486477-58487224- & 3.6635617 & 10.149518 & 0.0381737 & Up & Exonic & Lpar1 \\
\hline chr18:35610871-35613502+ & 2.0825754 & 10.667326 & 0.0465572 & Up & Exonic & Paip2 \\
\hline chr9:62912750-62923655- & 3.6162465 & 10.05098 & 0.0484927 & Up & Exonic & Pias1 \\
\hline chr19:38620443-38652111+ & 3.9124401 & 10.222612 & 0.0187912 & Up & Exonic & Plce1 \\
\hline chr4:103319022-103353651+ & 2.697894 & 10.275169 & 0.0383703 & Up & Exonic & Oma1 \\
\hline chr12:85028770-85034034+ & 3.3020582 & 10.391282 & 0.0454927 & Up & Exonic & Ylpm1 \\
\hline chr19:16705875-16710941- & 3.670584 & 10.055549 & 0.0445849 & Up & Exonic & Vps13a \\
\hline chr3:96041338-96042928- & 3.6635886 & 10.149032 & 0.0382528 & Up & Exonic & vps45 \\
\hline chr7:131074200-131081212+ & 3.6705236 & 10.056243 & 0.0443952 & $\mathrm{Up}$ & Exonic & Dmbt1 \\
\hline chr8:76908270-76910028+ & 3.6949691 & 10.054757 & 0.0436874 & Up & Exonic & $\mathrm{Nr} 3 \mathrm{c} 2$ \\
\hline chr3:133320936-133339349+ & 4.0277757 & 10.066357 & & Up & Exonic & $\mathrm{Ppa} 2$ \\
\hline $9-64108263+$ & 4.2291415 & 10.236954 & 358 & Up & Exonic & Pgm1 \\
\hline chr6:28668558-28724981+ & 3.903782 & 10.004567 & 0.0438149 & Up & Exonic & d 1 \\
\hline chr1:143667625-143677855- & 3.9271951 & 10.05 & 0.032 & Up & & \\
\hline chr2: & 3.629488 & 10.3 & & Up & & \\
\hline 040887-131046588+ & 2.7077757 & 10.28224 & 0.0367382 & Up & & \\
\hline 376803-109895641+ & 3.6595546 & 10.234915 & 034 & Up & & \\
\hline chr13:74671308-74675906+ & 3.9207004 & 10.345085 & 0.014267 & Up & & \\
\hline 3343-14140372- & 3.9823021 & 10.103344 & 0.0275104 & Up & & arf1 \\
\hline chr5:37 & 4.4134059 & 10.244003 & 0.0063616 & Up & & Ankib1 \\
\hline chr9:123175735-123190130- & 3.6655657 & 10.055021 & 0.0449695 & Up & Exonic & Cdcp1 \\
\hline chr6:83308933-83313546- & 3.9352621 & 10.00748 & 0.0422313 & Up & Exonic & Mthfd2 \\
\hline chr6:40819346-40829462+ & 3.6057348 & 10.049968 & 0.0493249 & Up & Antisense & Mir1950 \\
\hline r2:168705123-168710976- & 1.6193747 & 11.082478 & 0.0368852 & Up & Exonic & Atp9a \\
\hline $5: 72999972-73031643-$ & 3.30 & 214 & 0.0449857 & Up & Exonic & $\mathrm{c} 9$ \\
\hline CircRNAID & $\operatorname{logFC}$ & & & Regulation & IrcRNA Type & Gene Symbol \\
\hline chr14:17 & & & & Down & & Thrb \\
\hline & & 31 & & & & \\
\hline & 613 & 12.01 & 0.0 & & & \\
\hline & & 10.269 & 0. & & & in 1 \\
\hline & & 10.390823 & 0.0463646 & Down & Exonic & Man2a1 \\
\hline & 3.839478 & 1776 & 0.0200598 & Down & Exonic & \\
\hline & & & & & & \\
\hline 2885 - & 3.838912 & 10.05 & & Do & Exc & Pdlim5 \\
\hline $69-6$ & 4.294453 & 10.232286 & 0.0100338 & Down & Exonic & Upf2 \\
\hline $600+$ & 4.294953 & 10.4 & & Down & Exonic & Slc20a2 \\
\hline & 1.71 & 11. & & Down & e overlapping & Cwf1 1912 \\
\hline 9. & 3.54 & 10.34 & 0.033 & Down & Exonic & Cobl \\
\hline chr 5 & & 10 & & Do & Exonic & Ye: \\
\hline & 4.295033 & 10.268226 & 0.0087054 & Down & Exonic & Clasp1 \\
\hline $1756-93356079+$ & 3.839227 & 10.348148 & 0.019292 & Down & Exonic & Homer1 \\
\hline chr3:7 & 1.159965 & 11.770678 & 0.0496387 & Down & Exonic & Sis \\
\hline chr1 & & 10.135036 & 0.0344207 & Down & Exonic & Fam120a \\
\hline & 1.7 & 11.136284 & & Do & Exonic & Tmem245 \\
\hline & 4.084959 & 10.2 & & Down & Exonic & Fam188a \\
\hline $\mathrm{chr}$ & 4.642202 & & & & Exonic & Clip2 \\
\hline & & & & & & \\
\hline & & & & & Ex & Cnot4 \\
\hline & & & & Dc & Exonic & Morc3 \\
\hline & & & & & & Ankhd1 \\
\hline & & & & & & Phkb \\
\hline & & & & & & Sis \\
\hline & & & & & & \\
\hline & & & & & & \\
\hline $\mathrm{chr}$ & & & & & & 11 \\
\hline & & & & & & \\
\hline & & & & & & \\
\hline chr5:1 & 1.13 & 12. & & & Ex & \\
\hline & & 10.0 & & & Ex & \\
\hline & 1.7 & 11.3 & & $\mathrm{Dc}$ & Ex & a8 \\
\hline & 3.838528 & 10.14 & 12 & Do & & \\
\hline & $\begin{array}{l}4.086319 \\
2506461\end{array}$ & $\begin{array}{l}10.26 \\
10.91\end{array}$ & & $\begin{array}{l}\text { Do } \\
\text { Do }\end{array}$ & & p9 \\
\hline & & $\begin{array}{l}10.914739 \\
10.18655\end{array}$ & & $\begin{array}{l}\text { Do } \\
\text { Do }\end{array}$ & & R3hac1l \\
\hline $\begin{array}{l}\text { chr11: } \\
\text { chr1: }\end{array}$ & $\begin{array}{l}3.542513 \\
4295118\end{array}$ & $\begin{array}{l}10.186554 \\
1034959\end{array}$ & 435861 & & & \\
\hline 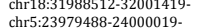 & $\begin{array}{l}4.295118 \\
2.839174\end{array}$ & $\begin{array}{l}10.349589 \\
1039375\end{array}$ & & Down & & \\
\hline $\begin{array}{l}38-24000019- \\
752-89483524-\end{array}$ & $\begin{array}{l}2.0372513 \\
1.772513\end{array}$ & $\begin{array}{r}10.39375 \\
11.067811\end{array}$ & $\begin{array}{l}0.0210191 \\
0.0454736\end{array}$ & Down & Exo & \\
\hline (201 & 4.085045 & 10.684208 & 0.0085185 & Dov & Exc & \\
\hline-115390436 & 3.839686 & 10.186561 & 0.027 & Down & $\begin{array}{l}\text { Intronic } \\
\text { Ind }\end{array}$ & $\mathrm{Zfc} 3 \mathrm{~h} 1$ \\
\hline r17:26266283-26267358+ & 3.839973 & 10.050 & 0.0406993 & Down & Exonic & Luc71 \\
\hline $18-55248 c$ & 3.541814 & 10.229497 & 0.0391764 & Down & Exonic & $\mathrm{Ns}$ \\
\hline chr9:60850130-60854503+ & 3.839332 & 10.095441 & 0.0369529 & Down & Exonic & Uaca \\
\hline : 149068050-149084 & 2.69038 & 10.496555 & 0.02675 & Down & verlapping & ennd5b \\
\hline r8:79343654-79372364- & 4.084385 & 10.050006 & 0.0298367 & Down & Exonic & \\
\hline
\end{tabular}


Fig. 2. Identification of circRNAs differentially expressed after exposure to radiation. (A) Expression of circRNAs in the control and irradiated group. (B) At day 3.5 post-radiation, 42 and 48 circRNAs were significantly upregulated and downregulated ( $\mathrm{p} \leq 0.05$, fold change $\geq 2$ ), respectively. (C) Overlapping number of targeted mRNAs of differentially expressed circRNAs and mRNAs.

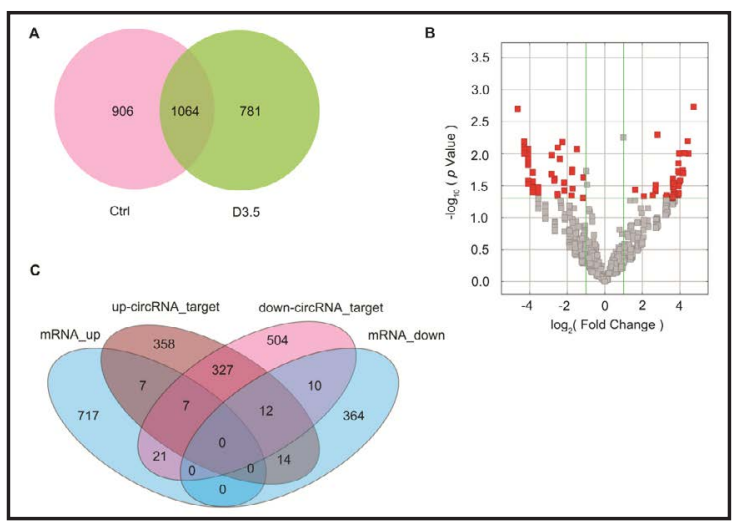

Fig. 3. Validation of circRNA expression by RT-PCR. Changes in expression were confirmed using RT-PCR for select circRNAs in the control and irradiated group. Bars represent mean $\pm \operatorname{SEM}(n=3)$, ** $\mathrm{P}<0.05$.

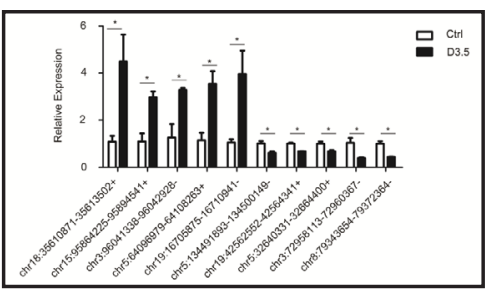

trends observed in the sequencing data.

\section{Functional analyses of miRNA target genes \\ To understand} the functions of genes associated with the differentially expressed circRNAs, GO analysis was performed. GO analysis included identification of molecular functions (Fig. 4A), biological processes (Fig. 4B), and cellular components (Fig. 4C). Of the molecular functions, the two main functions identified were binding and catalytic activity. The primary biological process identified was secretion, such as cytokine and cell secretion. When classified according to cellular component, the largest proportion of target genes was in the cell part and cell categories.

The functions of the predicted circRNA-target mRNAs were characterized by KEGG pathway analysis
Table 3. mRNA for GO and KEGG signaling pathway analyses

\begin{tabular}{|c|c|c|c|c|c|c|}
\hline Gene & Locus & D3.5_FPKM & Ctrl_FPKM & $\log 2(\mathrm{FC})$ & P Value & Regulation \\
\hline Celsr2 & chr3:108390850-108415552 & 0.798272 & 0.238657 & 1.74194 & 0.0006 & Up \\
\hline Trim6 & chr7:104218792-104235152 & 2.40461 & 0.989552 & 1.28095 & 0.00015 & Up \\
\hline Zfp37 & chr4:62189539-62208446 & 1.80806 & 0.883302 & 1.03346 & 0.00145 & Up \\
\hline Pex5l & chr3:32939313-33143247 & 0.649164 & 0.253207 & 1.35827 & 0.00455 & Up \\
\hline Tbc1d2 & chr4:46604389-46650209 & 2.87155 & 1.39098 & 1.04573 & 0.0001 & Up \\
\hline Steap2 & chr5:5664830-5694578 & 4.68727 & 2.28191 & 1.0385 & 0.00015 & Up \\
\hline Sfxn5 & chr6:85213050-85333422 & 0.945922 & 0.382691 & 1.30554 & 0.019 & Up \\
\hline Polr1e & chr4:45018582-45084604 & 4.29142 & 1.8369 & 1.22418 & 0.0023 & Up \\
\hline Lrp8 & chr4:107802258-107876840 & 1.18463 & 0.453724 & 1.38455 & 0.00175 & Up \\
\hline Pgap1 & chr1:54472999-54557684 & 9.3491 & 3.84237 & 1.28283 & 0.00005 & Up \\
\hline Gm4724 & chr2:175372027-175435807 & 1.23671 & 0.011015 & 6.81095 & 0.00545 & Up \\
\hline Slc1a2 & chr2:102658658-102790784 & 0.967178 & 0.223213 & 2.11536 & 0.00355 & Up \\
\hline Ephb2 & chr4:136647538-136835988 & 12.4825 & 5.80317 & 1.10499 & 0.00005 & Up \\
\hline Pappa & chr4:65124173-65357509 & 1.41139 & 0.703406 & 1.00469 & 0.00005 & Up \\
\hline Clec9a & chr6:129397296-129424764 & 0.573743 & 1.39147 & -1.27813 & 0.01185 & Down \\
\hline Serpinb8 & chr1:107590005-107608909 & 0.406235 & 0.984013 & -1.27636 & 0.01965 & Down \\
\hline Hpgds & chr6:65116828-65144908 & 0.976493 & 2.09466 & -1.10104 & 0.023 & Down \\
\hline $\mathrm{Cml} 2$ & chr6:85865421-85869158 & 1.03316 & 2.21287 & -1.09885 & 0.00005 & Down \\
\hline Gata3 & chr2:9857077-9890034 & 0.250508 & 0.720621 & -1.52439 & 0.0107 & Down \\
\hline Ikzf2 & chr1:69513931-69685960 & 0.451392 & 1.81571 & -2.00808 & 0.00005 & Down \\
\hline Zfp831 & chr2:174643533-174710832 & 0.188597 & 0.531756 & -1.49545 & 0.00005 & Down \\
\hline Heyl & chr4:123233555-123249875 & 0.208397 & 0.940518 & -2.17412 & 0.00005 & Down \\
\hline Cd53 & chr3:106758860-106790149 & 1.7224 & 4.4461 & -1.36812 & 0.00005 & Down \\
\hline Igj & chr5:88520511-88527891 & 127.153 & 421.247 & -1.7281 & 0.00005 & Down \\
\hline Sprr2a1 & chr3:92215834-92257298 & 13.981 & 38.726 & -1.46984 & 0.00005 & Down \\
\hline Srgap3 & chr6:112717970-112947266 & 0.428386 & 1.16302 & -1.44089 & 0.0004 & Down \\
\hline Cytip & chr2:58129136-58195532 & 4.25494 & 12.044 & -1.5011 & 0.00005 & Down \\
\hline Itga 4 & chr2:79255425-79461107 & 2.67814 & 6.40145 & -1.25717 & 0.00005 & Down \\
\hline Laptm5 & chr4:130913124-130936141 & 6.88928 & 15.8094 & -1.19835 & 0.00005 & Down \\
\hline Mras & chr9:99385421-99437376 & 0.753355 & 1.51864 & -1.01138 & 0.02225 & Down \\
\hline Cacna1e & chr1:154392518-154884037 & 0.39532 & 0.869295 & -1.13683 & 0.0002 & Down \\
\hline Kctd14 & chr7:97451333-97459553 & 3.52574 & 7.56646 & -1.10169 & 0.0078 & Down \\
\hline Prkcb & chr7:122288750-122634402 & 0.916544 & 3.11893 & -1.76677 & 0.00005 & Down \\
\hline Ptpn7 & chr1:135132724-135145326 & 0.471384 & 1.33024 & -1.49671 & 0.00005 & Down \\
\hline St6galnac3 & chr3:153205404-153725062 & 0.412624 & 1.12331 & -1.44486 & 0.009 & Down \\
\hline Cd84 & chr1:171839696-171890718 & 1.22365 & 2.52975 & -1.0478 & 0.0017 & Down \\
\hline
\end{tabular}

Table 4. mRNA involved signaling pathway

\begin{tabular}{|c|c|c|c|c|c|}
\hline $\begin{array}{l}\text { Pathway ID } \\
\end{array}$ & Definition & Fisher-PValue & Enrichment_Score & GeneRatio & Genes \\
\hline mmu04010 & $\begin{array}{l}\text { Kigsignaling pathway - } \\
\text { musculus (mouse) }\end{array}$ & 0.000887403 & 3.051879 & 0.285714 & Cacnale//Mras $/ / \mathrm{Prkcb} / / \mathrm{Ptpn} 7$ \\
\hline mmu05140 & Leishmaniasis - Mus musculus (mouse) & 0.005967734 & 2.224191 & 0.142857 & Itga 4//Prkcb \\
\hline mmu04724 & $\begin{array}{l}\text { Gilutamatergic synapse - Mus musculus } \\
\text { (moose) }\end{array}$ & 0.0181527 & 1.741059 & 0.142857 & Prkcb//slc1a2 \\
\hline mmu04670 & $\begin{array}{l}\text { Leukocyte transendothelial migration - } \\
\text { Mus musculus (mouse) }\end{array}$ & 0.01965651 & 1.706494 & 0.142857 & Itga4///Prkcb \\
\hline mmu04360 & Axon guidance - Mus musculus (mouse) & 0.02216906 & 1.654253 & 0.142857 & Ephb2P//Srgap3 \\
\hline mmu04530 & Tight junction - Mus musculus (mouse) & 0.02583172 & 1.587847 & 0.142857 & Mras//Prkcb \\
\hline mmu04020 & $\begin{array}{l}\text { Calcium signaling pathway - Mus } \\
\text { musculus (mouse) }\end{array}$ & 0.04145532 & 1.38242 & 0.142857 & Cacna1e//Prkcb \\
\hline
\end{tabular}


Fig. 4. Gene ontology analysis and KEGG signaling pathway analysis of selected mRNAs. Gene ontology analysis identified (A) molecular functions, (B) biological processes, and (C) cellular components. (D) Relevant pathways were identified for selected mRNAs.

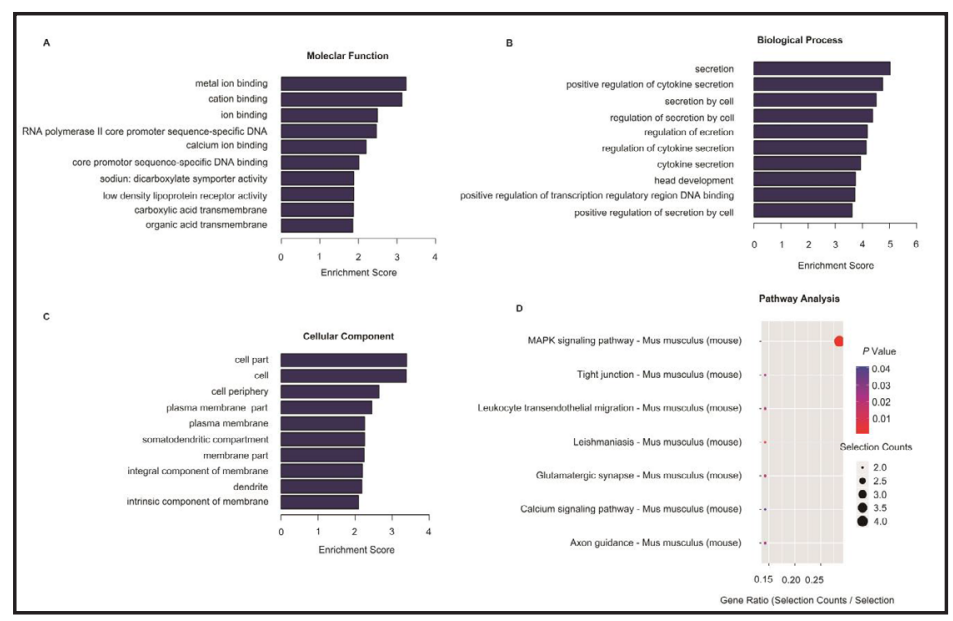

(Fig. 4D). The differentially expressed circRNA-target mRNAs were associated with the MAPK signaling pathway, leishmaniasis, glutamatergic synapses, leukocyte transendothelial migration, etc. For the MAPK signaling pathway (Table 4), four predicted mRNAs, Cacna1e, Mras, Prkcb, Ptpn7 were found to be involved in this pathway. Prkcb was also found to be involved in the calcium signaling pathway, tight junction leukocyte transendothelial migration, glutamatergic synapses, and leishmaniasis. It has been reported that EGF-mediated activation of the Ras/Raf/ Mek/Erk/MAPK signaling pathway is involved in intestinal stem cell proliferation [33] and more than half of mitosis by

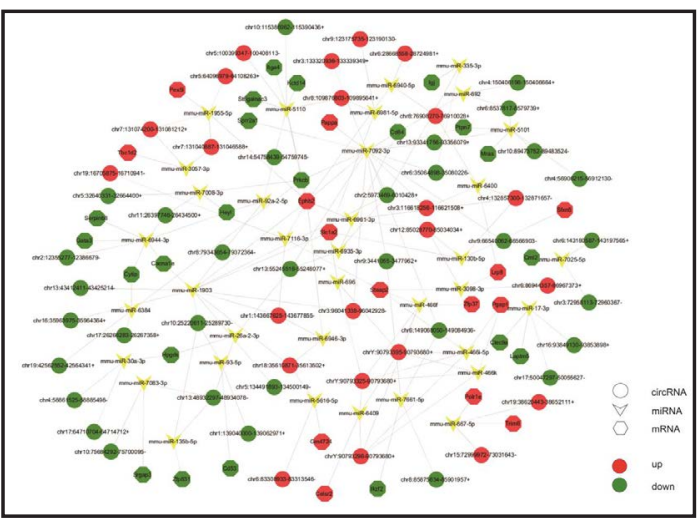

Fig. 5. Network of circRNA-miRNA-mRNA. Circles represent circRNAs, arrowheads represent miRNAs, hexagons represent mRNAs, red represents upregulated, and green represents downregulated. intestinal stem cells and transit amplifying progenitors relies on high levels of EGF within the crypt-domains [34]. Mihaescu [35] reported p38 MAPK is an important signaling pathway in radiation-induced colitis. Irradiation induces an increase in leukocyte and platelet recruitment, myeloperoxidase activity, CXC chemokine levels, and intestinal leakage. Inhibition of p38 MAPK decreases radiation-induced leukocyte and platelet recruitment.

\section{Prediction of miRNA binding sites and circRNA-miRNA-mRNA networks}

The selected 42 upregulated circRNAs with 14 associated targeted mRNAs and 48 downregulated circRNAs with 22 associated targeted mRNAs were used to construct a circRNA-miRNA-mRNA network (Fig. 5). The resulting network is very complicated, where one circRNA can interact with multiple miRNAs and one miRNA can inhibit multiple mRNAs. The miRNA mmu-miR-7092-3p interacted with the most circRNAs and mRNAs. Its target gene, Ephb2, is an intestinal stem cell marker and has been studied extensively in colorectal cancer [36]. The functions of circRNAs and their target genes in the intestine require additional studies in the future.

\section{Discussion}

In this present study, we performed a basic analysis of the functions of circRNAs associated with radiation-induced intestinal injury. Using sequencing technology, 2751 
circRNAs were identified from control and irradiated mice, where 1809 circRNAs had been previously identified and 942 were first observed in this study. Among the 2751 circRNAs, 1845 circRNAs were detected at 3.5 days post-radiation, which is the time point where the most severe intestinal injury is observed. The 2751 circRNAs were located across all the chromosomes, including the sex and mitochondrial chromosomes. These circRNAs were derived from a variety of regions, including exonic, sense overlapping, and intronic regions.

We selected 90 significantly regulated circRNAs and predicted their interacting miRNAs and miRNA-targeted mRNAs. For certain circRNAs, both the circRNA and their target mRNA(s) were upregulated or downregulated, as circRNAs function as competing endogenous RNAs and can interact with microRNAs through microRNA-response elements to cause miRNAinduced gene silencing. By comparing the prediction results with the mRNA sequencing results, 14 upregulated circRNA-targeted mRNAs and 22 downregulated circRNA-targeted mRNAs were identified. Based on their consistency of regulation, they may have greater chances of interacting.

Using the selected mRNAs, GO analysis was performed. It was found these mRNAs function mainly in the biological processes of cytokine and cell secretion, which may be associated with radiation-induced enteritis [37]. Some researchers have compared inflammatory bowel disease and normal segments of colon from patients with radiation proctitis against normal controls and found that radiation-induced tissue damage is associated with elevated levels of interleukins, especially interleukin-1 $\beta$ [38]. Perhaps these secretion processes are associated with increasing levels of interleukins.

KEGG signaling pathway analysis revealed the differentially expressed circRNA-target mRNAs are involved in the MAPK signaling pathway, leishmaniasis, glutamatergic synapses, and leukocyte transendothelial migration. As mentioned above, the MAPK signaling pathway is involved in intestinal stem cell proliferation [34] and radiation-induced colitis [35]. The third related pathway is leukocyte transendothelial migration, which is a key step in the inflammatory response. The KEGG signaling pathway analysis indicates that the differentially expressed circRNA-target mRNAs are mainly involved in the radiation-induced intestinal inflammatory response and intestinal stem cell regeneration.

The circRNA-miRNA-mRNA network is very complex, where one circRNA may have multiple miRNA binding sites and interact with multiple miRNAs. For example, CDR1as contains more than 70 binding sites for miR-7 and can also interact with miR-671 [3]. One miRNA can also interact with multiple circRNAs or one miRNA may play different roles by inhibiting different mRNAs. For example, in colon cancer, miR-7 inhibits the proliferation and migration of colon cancer cells by regulating FAK [39]. However, in the human colonic epithelial cell line LS174T, miR-7 binds to the 3' UTR of TFF3 to inhibit proliferation and migration via the PI3K-AKT signaling pathway [40]. Our comparison of circRNA-target mRNAs with sequencing mRNA results may facilitate screening for downstream targets.

More than $70 \%$ of cancer patients suffer from radiation therapy as the intestine is sensitive to radiation and is hard to shield. Treatments, such as hematopoietic stem cell transplantation and abdominal/pelvic tumor radiotherapy, injure the intestine to different degrees. Radiation-induced intestinal injury leads to intestinal mucosal injury, microbial translocation, inflammation, and, in severe cases, even death. Some researchers have reported methods of reducing structural damage to the intestine or promoting recovery of the intestinal structure after irradiation. However, there are currently no recognized approaches for the prevention and/or treatment of radiation-induced intestinal injury. The circRNA sequencing results in this study predicted that circRNAs are differentially expressed after irradiation and the miRNA and mRNA prediction results suggest that some mRNAs are involved in signaling pathways and biological functions with roles in the radiation-induced intestinal inflammatory response and intestinal stem cell regeneration. It is reasonable to conclude these differentially expressed circRNAs may play important roles in radiationinduced intestinal injury and/or repair.

In conclusion, our study is the first to delineate the expression profiles of circRNAs in mouse intestines after radiation. Abundant circRNAs were found in the jejuna of mice, where 
Lu et al.: Jejual CircRNAs Altereation After Radiation

some circRNAs were differentially expressed post-radiation. Interacting miRNAs were predicted for the differentially expressed circRNAs by analyzing the mRNA sequencing results, potential gene ontologies, and identifying involved signaling pathways. The sequencing results and predictions provide a resource for further mechanistic studies. However, the sponge function of circRNAs and their target mRNAs was predicted, this function needs to be experimentally verified. As there are currently no recognized methods of preventing and/or treating radiation-induced intestinal injury, the circRNA analysis performed in this present study may provide novel avenues for radiation-induced intestinal injury studies. We hope to identify circRNAs that help protect the intestine from irradiation that can be used or targeted to lessen intestinal injury from radiation therapy in the clinic.

\section{Acknowledgements}

This study was supported by the National Natural Science Foundation of China (31670859), CAMS Innovation Fund for Medical Science(2017-I2M-1-016),PUMC Youth Fund and the Fundamental Research Funds for the Central Universities(3332016100, 10023201601602), Research Funds for the Innovation Team of IRM-CAMS (1650).

\section{Disclosure Statement}

The authors declare to have no competing interests.

\section{References}

1 Danan M, Schwartz S, Edelheit S, Sorek R: Transcriptome-wide discovery of circular RNAs in Archaea. Nucleic Acids Res 2012;40:3131-3142.

-2 Memczak S, Jens M, Elefsinioti A, Torti F, Krueger J, Rybak A, Maier L, Mackowiak SD, Gregersen LH, Munschauer M, Loewer A, Ziebold U, Landthaler M, Kocks C, le Noble F, Rajewsky N: Circular RNAs are a large class of animal RNAs with regulatory potency. Nature 2013;495:333-338.

-3 Piwecka M, Glažar P, Hernandez-Miranda LR, Memczak S, Wolf SA, Rybak-Wolf A, Filipchyk A, Klironomos F, Cerda Jara CA, Fenske P, Trimbuch T, Zywitza V, Plass M, Schreyer L, Ayoub S, Kocks C, Kühn R, Rosenmund C, Birchmeier C, Rajewsky N: Loss of a mammalian circular RNA locus causes miRNA deregulation and affects brain function. Science 2017;357:eaam8526.

-4 Zhang XO, Wang HB, Zhang Y, Lu X, Chen LL, Yang L: Complementary sequence-mediated exon circularization. Cell 2014; 159: 134-147.

5 Jeck WR, Sorrentino JA, Wang K, Slevin MK, Burd CE, Liu J, Marzluff WF, Sharpless NE: Circular RNAs are abundant, conserved, and associated with ALU repeats. RNA 2013;19:141-157.

6 Zhang Y, Zhang XO, Chen T, Xiang JF, Yin QF, Xing YH, Zhu S, Yang L, Chen LL: Circular intronic long noncoding RNAs. Mol Cell 2013; 51: 792-806.

7 Lasda E, Parker R: Circular RNAs: diversity of form and function. RNA 2014;20:1829-1842.

8 Salzman J, Gawad C, Wang PL, Lacayo N, Brown PO: Circular RNAs Are the Predominant Transcript Isoform from Hundreds of Human Genes in Diverse Cell Types. PLoS One 2012;7:e30733.

-9 Pamudurti NR, Bartok O, Jens M, Ashwal-Fluss R, Stottmeister C, Ruhe L, Hanan M, Wyler E, PerezHernandez D, Ramberger E, Shenzis S, Samson M, Dittmar G, Landthaler M, Chekulaeva M, Rajewsky N, Kadener S: Translation of CircRNAs. Mol Cell 2017;66:9-21.e27.

-10 Nitsche A, Doose G, Tafer H, Robinson M, Saha NR, Gerdol M, Canapa A, Hoffmann S, Amemiya CT, Stadler PF: Atypical RNAs in the coelacanth transcriptome. J Exp Zool B Mol Dev Evol 2014;322:342-351.

-11 Hansen TB, Jensen TI, Clausen BH, Bramsen JB, Finsen B, Damgaard CK, Kjems J: Natural RNA circles function as efficient microRNA sponges. Nature 2013;495:384.

12 Li Z, Huang C, Bao C, Chen L, Lin M, Wang X, Zhong G, Yu B, Hu W, Dai L, Zhu P, Chang Z, Wu Q, Zhao Y, Jia Y, Xu P, Liu H, Shan G: Exon-intron circular RNAs regulate transcription in the nucleus. Nat Struct Mol Biol 2015;22: 256-264. 


\section{Cellular Physiology Cell Physiol Biochem 2018;47:2558-2568

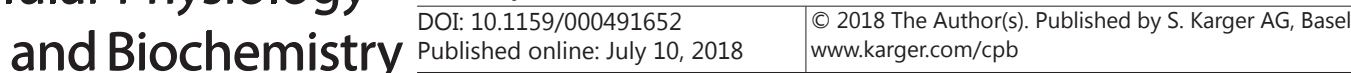

Lu et al.: Jejual CircRNAs Altereation After Radiation

13 Lukiw WJ: Circular RNA (circRNA) in Alzheimer's disease (AD). Front Genet 2013;4: 307.

14 Bachmayr-Heyda A, Reiner AT, Auer K, Sukhbaatar N, Aust S, Bachleitner-Hofmann T, Mesteri I, Grunt TW, Zeillinger R, Pils D: Correlation of circular RNA abundance with proliferation-exemplified with colorectal and ovarian cancer, idiopathic lung fibrosis, and normal human tissues. Sci Rep 2015;5:8057.

15 Shang X, Li G, Liu H, Li T, Liu J, Zhao Q Wang C: Comprehensive circular RNA profiling reveals that hsa circ_0005075, a new circular RNA biomarker, is involved in hepatocellular carcinoma development. Medicine (Baltimore) 2016; 95:e3811.

16 Li P, Chen S, Chen H, Mo X, Li T, Shao Y, Xiao B, Guo J: Using circular RNA as a novel type of biomarker in the screening of gastric cancer. Clin Chim Acta 2015;444:132-136.

17 Hauer-Jensen M, Denham JW, Andreyev HJN: Radiation Enteropathy-Pathogenesis, Treatment, and Prevention. Nature reviews. Nat Rev Gastroenterol Hepatol 2014;11:470-479.

18 Thomas GM: Concurrent chemotherapy and radiation for locally advanced cervical cancer: the new standard of care. Sem Radiat Oncol 2000;10:44-50.

19 Mettler FAJ, Voelz GL: Major Radiation Exposure -What to Expect and How to Respond. N Engl J Med 2002;346:1554-1561.

20 Berger ME, Christensen DM, Lowry PC, Jones OW, Wiley AL: Medical management of radiation injuries: current approaches. Occup Med (Oxf) 2006;56:162-172.

21 Zhang H, Yan H, Zhou X, Wang H, Yang Y, Zhang J, Wang H: The protective effects of Resveratrol against radiation-induced intestinal injury. BMC Complement Altern Med 2017;17:410.

22 Macià i Garau M, Lucas Calduch A, López EC: Radiobiology of the acute radiation syndrome. Rep Pract Oncol Radiother 2011;16:123-130.

23 Monti P, Wysocki J, van der Meeren A, Griffiths NM: The contribution of radiation-induced injury to the gastrointestinal tract in the development of multi-organ dysfunction syndrome or failure. BJR Suppl 2005;27:89-94.

24 Gong W, Guo M, Han Z, Wang Y, Yang P, Xu C, Wang Q, Du L, Li Q, Zhao H, Fan F, Liu Q: Mesenchymal stem cells stimulate intestinal stem cells to repair radiation-induced intestinal injury. Cell Death Dis 2016;7:e2387.

25 Liang Y, Zhou H, Yao Y, Deng A, Wang Z, Gao B, Zhou M, Cui Y, Wang L, Zhou L, Wang B, Wang L, Liu A, Qiu L, Qian K, Lu Y, Deng W, Zheng X, Han Z, Li Y, Sun J: 12-O-tetradecanoylphorbol-13-acetate (TPA) increases murine intestinal crypt stem cell survival following radiation injury. Oncotarget 2017;8:45566-45576.

26 Jang H, Park S, Lee J, Myung Jk, Jang WS, Lee SJ, Myung H, Lee C, Kim H, Lee SS, Jin YW, Shim S: Rebamipide alleviates radiation-induced colitis through improvement of goblet cell differentiation in mice. J Gastroenterol Hepatol 2018;33:878-886.

27 Han NK, Jeong YJ, Pyun BJ, Lee YJ, Kim S-H, Lee HJ: Geranylgeranylacetone Ameliorates Intestinal Radiation Toxicity by Preventing Endothelial Cell Dysfunction. Int J Mol Sci 2017;18:2103.

28 Martin M: Cutadapt removes adapter sequences from high-throughput sequencing reads. EMBnetjournal 2011;17:10-12.

29 Dobin A, Davis CA, Schlesinger F, Drenkow J, Zaleski C, Jha S, Batut P, Chaisson M, Gingeras TR: STAR: ultrafast universal RNA-seq aligner. Bioinformatics 2013;29:15-21.

-30 Cheng J, Metge F, Dieterich C: Specific identification and quantification of circular RNAs from sequencing data. Bioinformatics 2016;32:1094-1096.

-31 Glažar P, Papavasileiou P, Rajewsky N: circBase: a database for circular RNAs. RNA 2014;20:1666-1670.

-32 Robinson MD, McCarthy DJ, Smyth GK: edgeR: a Bioconductor package for differential expression analysis of digital gene expression data. Bioinformatics 2010;26:139-140.

-33 Cui S, Chang PY: Current understanding concerning intestinal stem cells. World J Gastroenterol 2016;22:7099-7110.

-34 Binnerts ME, Kim KA, Bright JM, Patel SM, Tran K, Zhou M, Leung JM, Liu Y, Lomas WE, Dixon M, Hazell SA, Wagle M, Nie WS, Tomasevic N, Williams J, Zhan X, Levy MD, Funk WD, Abo A: R-Spondin1 regulates Wnt signaling by inhibiting internalization of LRP6. Proc Natl Acad Sci U S A 2007;104:14700-14705.

-35 Mihaescu A, Santen S, Jeppsson B, Thorlacius H: p38 Mitogen-activated protein kinase signalling regulates vascular inflammation and epithelial barrier dysfunction in an experimental model of radiation-induced colitis. Br J Surg 2010;97:226-234.

-36 Jang BG, Kim HS, Kim KJ, Rhee YY, Kim WH, Kang GH: Distribution of intestinal stem cell markers in colorectal precancerous lesions. Histopathology 2016;68:567-577. 
Lu et al.: Jejual CircRNAs Altereation After Radiation

37 Anwar M, Ahmad S, Akhtar R, Mahmood A, Mahmood S: Antioxidant Supplementation: A Linchpin in Radiation-Induced Enteritis. Technol Cancer Res Treat 2017;16:676-691.

38 Indaram AVK, Visvalingam V, Locke M, Bank S: Mucosal cytokine production in radiation-induced proctosigmoiditis compared with inflammatory bowel disease. Am J Gastroenterol 2000;95:1221-1225.

-39 Zeng C, Zhan Y, Huang J, Chen Y: MicroRNA-7 suppresses human colon cancer invasion and proliferation by targeting the expression of focal adhesion kinase. Mol Med Rep 2016;13:1297-1303.

-40 Guo J, Xu L, Teng X, Sun M: MicroRNA-7-5p regulates the proliferation and migration of intestinal epithelial cells by targeting trefoil factor 3 via inhibiting the phosphoinositide 3-kinase/Akt signalling pathway. Int J Mol Med 2017;40:1435-1443. 